Revue d'histoire de l'Amérique française

DE REVUE D.HISTOIRE DE L'AMÉRIQUE FRANÇAISE

\title{
Index des auteurs et des ouvrages philosophiques au Séminaire de Québec (1792-1881)
}

Marc Lebel

Volume 19, numéro 2, septembre 1965

URI : https://id.erudit.org/iderudit/302486ar

DOI : https://doi.org/10.7202/302486ar

Aller au sommaire du numéro

Éditeur(s)

Institut d'histoire de l'Amérique française

ISSN

0035-2357 (imprimé)

1492-1383 (numérique)

Découvrir la revue

Citer ce document

Lebel, M. (1965). Index des auteurs et des ouvrages philosophiques au Séminaire de Québec (1792-1881). Revue d'histoire de l'Amérique française, 19(2), 323-328. https://doi.org/10.7202/302486ar d'utilisation que vous pouvez consulter en ligne.

https://apropos.erudit.org/fr/usagers/politique-dutilisation/ 


\title{
BIBLIOGRAPHIE
}

\section{INDEX DES AUTEURS ET DES OUVRAGES PHILOSOPHIQUES AU SÉMINAIRE DE QUÉBEC *}

\author{
1792-1881
}

\author{
APPENDICE A \\ Index des auteurs et des ouvrages philosophiques inscrits \\ au catalogue de l'abbé Dudevant (1782).
}

1. Dans la section "philosophie":

Aristoteles, Opera.

Arnaud et Nicole, La logique de Port-Royal ou l'art de penser.

Barbay, Commentarius in Aristotelis logicam, metaphysicam, moralem, physicam...

Bayle, Oeuvres.

Boyvin, Philosophia scoti a prolixitate, et subtilitas ejus ob obscuritate libera et vindicata...

Camier et Gigot, Institutiones philosophiax ad usum Seminarii Tullensis...

Dagoumer, Philosophia ad usum scholæ accommodata...

Du Hamel, Philosophia vetus et nova ad usum scholæ accommodate...

-, Philosophia universalis sive commentarius in universam Aristotelis philosophicam ad usum scholarum comparatum...

Duhan, Philosophus in utramque partem, sive selectæ et limitæ difficultates in utramque partem...

Galtruche, Institutiones philosophiar ...

Genest, Principes de philosophie ou preuves naturelles de l'existence de Dieu et de l'immortalité de l'âme.

Huet, Censura philosophiax cartesianx.

Leibnitz, Essai de théodicée.

Locke, Essay.

Malebranche, Entretiens de la métaphysique.

$\rightarrow$ Recherche de la vérité.

Olivet, La philosophie du bon sens ou réflexions philosophiques sur l'incertitude des connaissances humaines à l'usage des Cavaliers et du beau sexe...

* S.v.p. se reporter aux précédents articles de M. Lebel, notre Revue, XVIII : 405-424, 463-474, 582-593; XIX: 106-125, 238-253. 
Pluche, Histoire du ciel, où l'on recherche l'origine de l'idolâtrie et des méprises de la philosophie, sur la formation des corps célestes, et de toute la nature.

-, Spectacles de la nature, ou Entretiens sur l'histoire naturelle et les sciences.

Pourchot, Institutiones philosophicx ad faciliorem veterum ac recentiorum philosophicorum lectionem comparatx...

Rousseau, Oeuvres.

Voltaire, Oeuvres.

-, Dictionnaire philosophique.

-, Philosophie de Newton.

Ouvrages dont l'auteur n'est pas indiqué:

Aristotelis philosophia.

Commentaria in Aristotelis metaphysica.

Métaphysique (manuscrit).

Morale de Confucius, la morale de la Chine.

Manière de penser.

Specimena philosophix Descartes.

Totius philosophiæ principia.

2. Dans la section "livres de classe" 1 :

Aristotelis Stagiritio.

Compendium philosophiæ (manuscrit).

Definitiones philosophix.

Erasmi colloquia.

Philosophia sacra.

Philosophie de Goudin

(Philosophia juxta inconcussa tutissimaque Divi Thomæ dogmata)

Philosophie de Du Hamel.

Philosophia moralis.

Philosophia universalis.

\section{APPENDICE B}

Index des auteurs et des ouvrages philosophiques figurant sur la liste des livres vraisemblablement achetés par M. Holmes pour le Séminaire de Québec en 1836-37.

Ancillon, Histoire de la philosophie.

Barruel, Helviennes.

Bautain, Philosophie du christianisme.

Bergier, Certitudes des preuves du christianisme.

-, Examen du matérialisme.

-, Déisme réfuté.

-, Traité de la vraie religion.

- Dictionnaire de Théologie.

Blanchard, Ecole des mours.

Bouvier, Institutiones philosophiar.

1 C'est le titre même d'une section du catalogue. 
Condillac, Etudes élémentaires de philosophie.

De Bonald, Oeuvres complètes.

Duvoisin, Défense de l'ordre social.

Feller, Catéchisme philosophique.

Fénelon, Vie des philosophes.

Frayssinous, Conférences sur la religion.

Gérard, Eléments de philosophie.

Gerbet, Doctrine philosophique sur la certitude.

Lacordaire, Réfutation du système de M. de La Mennais.

Lamennais, Oeuvres complètes.

Laurentie, Introduction à la philosophie.

-, Methodus philosophandi.

Rosbeck, Essai sur l'action du philosophisme et du christianisme.

Savérien, Vie des philosophes modernes.

-, Philosophes anciens.

\section{APPENDICE C}

Index des ouvrages philosophiques inscrits au catalogue de la bibliothèque des écoliers (vers 1838).

Abrégé de la vie des philosophes (Fénelon ?).

Anti-dictionnaire philosophique (Nonnotte).

Catéchisme philosophique (Feller).

Conférences: (Frayssinous).

Dissertations: La Luzerne.

Helviennes (Barruel).

Institutions philosophiques.

Logique: Condillac.

(Oeuvres) : de Bonald.

Philosophes bienfaisants.

Philosophes chrétiens.

Portefeuille d'un philosophe chrétien.

Pratiques: Buffier.

\section{APPENDICE D}

Index des auteurs et des ouvrages cités dans les Institutiones philosophicæ ad usum studiosæ juventutis. (1835) ${ }^{1}$

Annales de philosophie chrétienne, 12-13, 152, 157-158, 168.

Antommarchi, F., Mémoires du docteur F. Antommarchi, 144.

Barclai, G., De regno et regali potestate, 333-335.

Barruel, Les Helviennes, 109-110, 111, 111-112, 116, 116-117, 117-118, 208$210,229,250-251,254-255,256$.

Bayle, Oeuvres, 216.

Beudant, Traité élémentaire de physique, 127.

Bergier, Traité de la religion, 125, 139, 139-140, 151-152, 226.

-, Dictionnaire de théologie, 207.

Blanchard, L'Ecole des mours, 142-143.

${ }^{1}$ La pagination renvoie au manuel de l'abbé Demers. 
Bonald (de), La législation primitive, 5, 11, 11-12, 17, 18, 25-26, 114, 118, $119,147-148,325$.

-, Recherches philosophiques, 5-6, 9-10, 10-11, 12, 17, 19, 22, 23, 25, 26, 26-27, 253.

Bossuet, 277.

-, Politique tirée de l'écriture sainte, 333.

Bouvier, Institutiones philosophica, 6, 79-80.

Brébeuf, 30.

Buffon, Discours sur les variétés de l'espèce humaine, 158-159.

Bussy-Rabutin, Lettre à $M$. de la Roquette, évêque d'Autun, 300-301.

Cabanis, Rapport du physique et du moral de l'homme, 232.

Champollion-Fegeac, 174-175.

Condillac, Logique, 9, 24.

-, Traité des sensations, 9.

Cousin-Despréaux, Leçons de la nature, 247, 248-249.

Cuvier, Recherches sur les ossements fossiles, 156.

-, Tableau élémentaire de l'histoire naturelle, 159, 169.

Du Clot, La Bible vengée, 150, 172, 212-213.

Duvoisin, Défense de l'ordre social, 316, 317, 319-320, 321, 322, 329, 330.

Encyclopédie (l'), 222.

Feller, Catéchisme philosophique, 240.

Fénelon, Traité de l'existence de Dieu, 135.

Fontenelle, 7-8.

Frayssinous, Conférences, 65, 72-73, 74, 88-89, 93-94, 113, 122-123, 124-125, 126-127, 154-157, 170, 230, 232-233, 234-235, 288, 290-291, 294, 295-297, 298, 298-299, 322-323, 324 .

Gérard, Essai sur les vrais principes, 135, 246.

-, Théorie du bonheur, 237-238.

Gerdil, Caractères distinctifs de l'homme et des brutes, 115.

Goguet, Origines des lois, 176.

Grégoire XVI, Singulari nos, 59.

Hauchecorne, Abrégé de philosophie, 14.

Holbac (d'), Système de la nature, 237.

Lacépède, Histoire naturelle de l'homme, 160-161.

Lamennais, Essai sur l'indifférence en matière de religion, (T. I, II) 58 , $112,285,290,316,323-326$.

-, Défense de l'essai sur l'indifférence en matière de religion, $57,58$.

Las Cases, Atlas historique, généalogique, chronologique et géographique, $152-154$.

La France de Pompignon, L'incrédulité convaincue par les prophéties, 199-200.

Le François, Preuves de la religion, 138.

Lucrèce, 116.

Lucain, 30.

Malebranche, 17.

Malte-Brun, Traité élémentaire de géographie, 318.

Montazet, Instruction pastorale sur les sources de l'incrédulité et sur les fondements de la religion, 92.

Montbrion, De la religion des Hébreux et de leur cosmogonie, 159-160.

Montesquieu, L'Esprit des lois, 149.

Para du Phanjas, Philosophie de la religion, 173.

Plutarque, 109.

Porphyre, 113. 
Rousseau, 25, 141, 143-144, 236, 300.

-, l'Emile, 179, 207-208, 215, 279-280.

- La Nouvelle Héloïse, 302-303, 305, 306.

-, Le contrat social, $320,321$.

-, Discours sur l'origine et les fondements de l'inégalité parmi les hommes, 24.

Racine (Louis), Poème de la religion, 113.

Valmont de Bomare, Dictionnaire d'histoire naturelle, 159.

Virey, Nouveau dictionnaire d'histoire naturelle, 129-130, 133, 134, 168, 169, $242,244-245,308-309,310$.

-, Histoire du genre humain, 161-162.

Voltaire, 120.

-, Dictionnaire philosophique, 147.

\section{APPENDICE E}

Index des auteurs et des ouvrages philosophiques inscrits au catalogue de la bibliothèque des écoliers en 1881 .

André, Essai sur le beau.

Anonyme, Don Quichotte philosophe.

-, Philosophe bienfaisant.

-, Philosophe chrétien.

-, Philosophes des trois premiers siècles.

-, Philosophicix prælectiones.

-, Platon polichinelle.

- Portefeuille d'un philosophe chrétien.

Balmes, Mélanges philosophiques et religieux.

Barbay, De logica Aristotelis.

-, Commentaria in Aristotelis physicam.

Barbe, Cours élémentaire de philosophie.

- Histoire de la philosophie.

Barruel, Helviennes.

-, Mémoires.

Bautain, Philosophie morale.

-, Psychologie expérimentale.

- L La religion et la liberté.

Bensa, Manuel de logique.

Bergier, Dictionnaire philosophique.

-, Dictionnaire théologique.

Bozzeli, Union de la morale.

Bonald (de), De l'opposition dans le gouvernement.

Buffier, Traité des premières vérités.

Chastel, Origine des connaissances humaines.

-, Rationalistes et traditionalistes.

Cousin-Despréaux, Leçons de la nature.

Decker, Cours élémentaire de philosophie.

Degerando, Histoire des systèmes philosophiques.

Delalle, Eléments de philosophie chrétienne.

Doney, Philosophica institutiones.

Feller, Catéchisme philosophique.

Fénelon, Philosophes de l'antiquité. 
Frayssinous, Défense du christianisme.

Frère, Philosophie de l'histoire.

Goudin, Philosophie.

Gratry, Philosophie.

-, Sophistique contemporaine.

$\rightarrow$ Logique.

Gravesande, Introduction à la philosophie.

Lebrethon, Somme théologique de saint Thomas d'Aquin.

Liberatore, Institutiones philosophica.

Maistre (de), Examen de la philosophie de Bacon.

Maret, Panthéisme.

Nicolas, Etudes philosophiques.

Nonnotte, Dictionnaire anti-philosophique.

Pluche, Spectacle de la nature.

Pradié, Philosophe...

Rattier, Manuel de philosophie.

Receveur, Essai sur la nature de l'âme.

Thomas d'Aquin (saint), Somme théologique.

Tongiorgi, Institutiones philosophicæ.

Ubaghs, Ontologia elementa.

-, Philosophia elementa.

-, Philosophicæ institutiones.

-, Philosophiaæ anthropologicæ.

Ventura, Conférences. 